\title{
Flash-Boiling Atomization of Liquids under Tensile Conditions
}

\author{
Tali Bar-Kohany*1,2, Eran Sher ${ }^{3}$ \\ ${ }^{1}$ School of Mechanical Engineering, Tel-Aviv University, Tel Aviv, 6997801, Israel \\ ${ }^{2}$ Department of Mechanical Engineering, nrcn, Beer-Sheva, 8419001, Israel \\ ${ }^{3}$ Faculty of Aerospace Engineering, Technion-Israel Institute of Technology, Haifa, Israel \\ *Corresponding author email: talibk@tauex.tau.ac.il
}

\begin{abstract}
When a flash boiling atomization is considered, a higher-pressure difference across the atomization unit (higher upstream or lower downstream pressures) is expected to result in a finer spray. Here we study, in theoretical terms, the effect of lowering the downstream pressure to its value at near the spinodal, well below the absolute zero (a negative pressure). A negative pressure in a continuous liquid phase can be accomplished when outward forces are applied to produce tensile stresses within the liquid. This state is a metastable liquid state.

The negative pressure is a new emerging technology that is already being used in some applications from drug and DNA delivery, gene therapy by sonoporation, tissue ablation (histotripsy), nanoparticles generation by sonochemistry, nuclear material detection and monitoring, to synthetic trees to capture carbon-dioxide by using hydro-gels and reverse osmosis.

Here we evaluate the bubbles' growth rate and their size at burst time as a result of homogeneous nucleation under negative pressure conditions. Further we stress that the bubbles' size at burst is a reasonable indication of the droplets' size and uniformity. We also examine the effect of the pressure negativity on the efficiency of transforming the input energy to the bubbles' energy at burst. We conclude that the negative downstream pressure does not necessarily contribute to obtain smaller droplets' or higher efficiency of the atomization process.
\end{abstract}

\section{Keywords}

Flash boiling atomization, Negative pressure, homogeneous nucleation.

\section{Introduction}

Evidence of negative pressure in liquids is dated back to the 19th century, with measurements of Marcellin Berthelot (1850) and of Worthington (1892), as stated in the excellent review by Caupin et. al. [1]. The existence of a negative pressure region is depicted also by any of the cubic equations of state (EoS). According to the Van der-Waals EoS, for example, negative pressure could be obtained for temperature of about $85 \%$ of the critical temperature, namely for a wide span of temperatures. For water, it means that negative pressure could be obtained for temperature values even lower than $273^{\circ} \mathrm{C}$. Water is important to almost every aspect in life, including trees [3] and medical applications [5]. Water-glass systems are expected to produce strong adhesion, due to the polarity of water and thanks to the polar $\mathrm{SiOH}$ groups of the glass [4]. Strong adhesion forces promote the ability of a liquid to sustain negative pressure. Indeed, most of the experiments and theoretical analysis performed for negative pressure in liquids is concerned with water. If so, the question arises whether nonpolar liquids can sustain negative pressure as well. Caupin et al. [5] conducted experiments on heptane, which is a non-polar liquid and found that it too, can sustain negative pressure. In their experiments, they reached more then $-20 \mathrm{MPa}$ at about $0{ }^{\circ} \mathrm{C}$. In their paper, they found similar values for ethanol, which is one of the main components in biofuels. More organic liquids were 
found to sustain negative pressure as early as in the 1950's by Briggs [6]. Hence, the ability to form an efficient spray is crucial to a viable application of these fuels.

Like water, negative pressure can be obtained at relatively high temperatures. Experimental evidence of negative pressure was obtained at around $200^{\circ} \mathrm{C}$ for heptane and ethanol. It is important to note that negative pressure is obtained for these liquids only when they are within the metastable region (see Figure 1); namely when the liquids are kinetically, rather than thermodynamically stable.

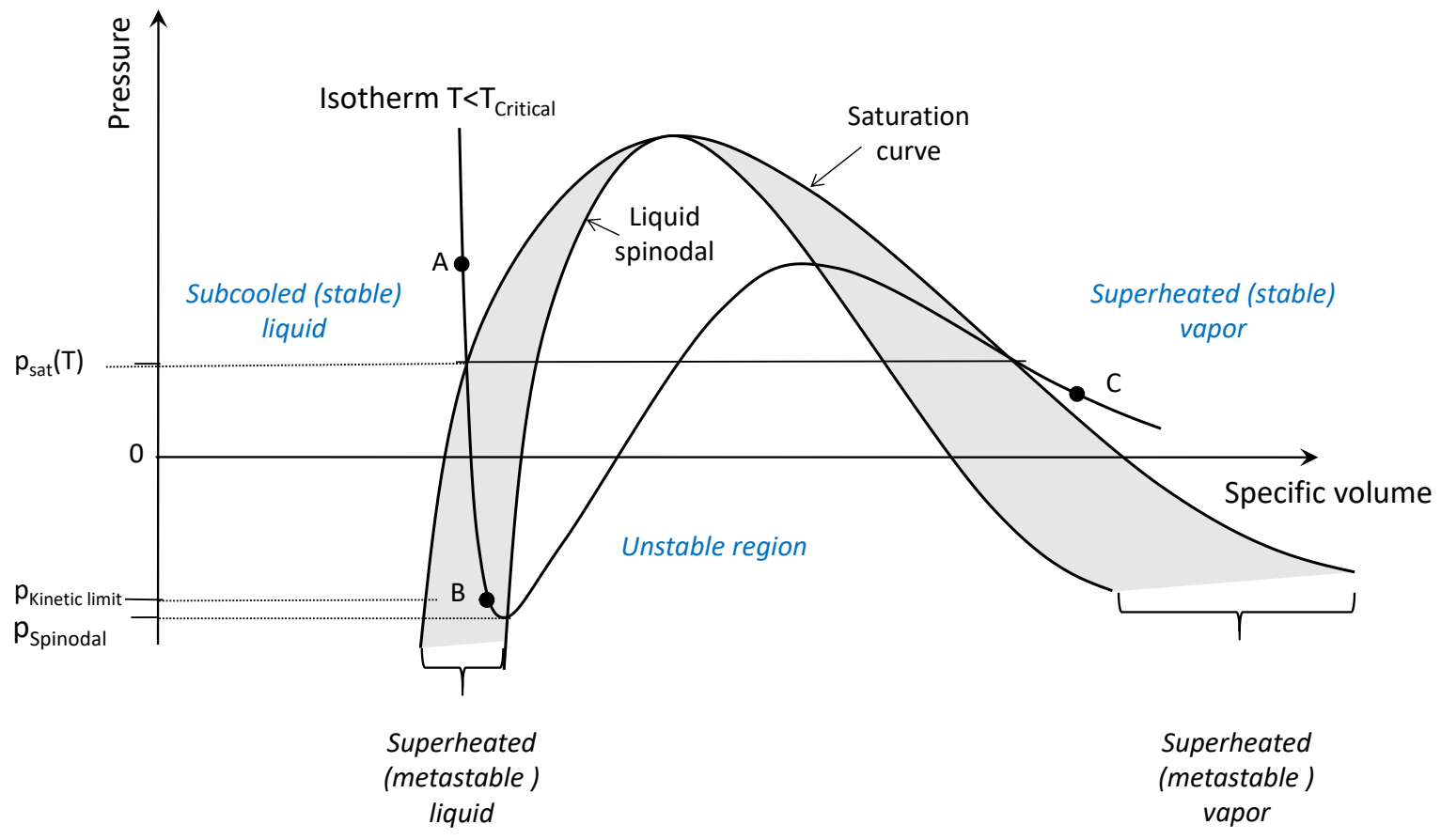

Figure 1. Saturation curve and spinodal line (adapted from [7]).

\section{Problem description and analysis}

We consider here a liquid, initially under compressed liquid conditions (point $A$ in Figure 1), that is depressurized isothermally to the kinetic limit (point $B$ in Figure 1), and as a result, homogeneous nucleation at constant pressure occurs. We choose to study isotherms that allow for a negative pressure to be reached near the spinodal, and as aforementioned, this domain is quite wide. Near the spinodal, nucleation is extremely vigorous [8]. At the very end of the process, when the bubbles touch each other (void fraction of about 0.5 [9]), small droplets are formed through various mechanisms [10].

It is well known that for sprays that are formed through heterogeneous nucleation, higher degrees of superheating, which are manifested by lower ambient pressure, result in lower mean diameter [11]. In the present paper, we will examine homogeneous nucleation; we will investigate bubbles' growth rate, and their size at burst time as a plausible indication of the droplets' size. The basic equations of state (EOS) that were developed originally for positive pressure values, can be used for liquids under negative pressure values (tensioned liquid), as is clearly stated by Carey (p.178 [13]).

We also present how the efficiency of this process depends on the pressure negativity. From the moment nucleation incepts, where the nuclei radius is $r_{c}$, they grow and at the same time new nuclei form. At time $\tau$, their volume is evaluated by their growth rate $(\dot{r})$ and the relative time of growth $(\tau-t)$. The void fraction $(\varepsilon)$ of a unit volume of the mixture is defined as: 


$$
\begin{aligned}
& \varepsilon \equiv\left(\frac{V_{b}}{V_{l}}\right)=\int_{0}^{\tau} \frac{4}{3} \pi\left\{\left[r_{c}+\left(\frac{d r}{d t}(\tau-t)\right)\right]^{3} \frac{d n}{d t}\right\} d t \\
& \dot{r} \equiv \frac{d r}{d t}=\sqrt{\frac{2 \Delta p}{3 \rho_{l}}} ; \Delta p=p_{s}-p
\end{aligned}
$$

Where $V_{b}$ and $V_{l}$ are the total bubbles and liquid volumes, respectively. $(\tau-t)$ is the period between the nucleation of a new set of nuclei to the time they've reached a radius $\mathrm{r}$. At time $\tau$, we designated the void fraction to be maximal $(\varepsilon=0.5)$.

The critical nuclei radius is negligibly small as compared to their radius at time $\tau$, where maximal void fraction prevails, and thus $\tau$ can be easily extracted:

$$
\tau=\left[\frac{3}{\pi} \frac{\varepsilon}{\dot{n}(\dot{r})^{3}}\right]^{1 / 4}
$$

The Sauter Mean Diameter (SMD) of the bubbles at that time is evaluated by the following expression. Bearing in mind that we are considering a constant pressure process, which is also very close to isothermal one [12], the nucleation rate can be considered constant $(\dot{n}=d n / d t=$ const $)$.

$$
(S M D)_{b}=\frac{\left(V_{b} / V_{l}\right)_{@ \tau}}{\left(A_{b} / V_{l}\right)_{@ \tau}}=\frac{\frac{4}{3} \pi \int_{0}^{\tau}\left\{\left[r_{c}+\left(\frac{d r}{d t}(\tau-t)\right)\right]^{3} \frac{d n}{d t}\right\} d t}{4 \pi \int_{0}^{\tau}\left\{\left[r_{c}+\left(\frac{d r}{d t}(\tau-t)\right)\right]^{2} \frac{d n}{d t}\right\} d t}=\frac{\varepsilon}{\frac{4 \pi}{3} \dot{n} \dot{r}^{2} \tau^{3}}=\frac{\dot{r} \tau}{4}
$$

The efficiency $(\eta)$ of the primary spray process can be defined as the ratio between the surface energy of bubbles at the bursting time and the work that is required to maintain a constant pressure in the liquid. The efficiency of the process in terms of droplets formation, rather than bubbles formation should be merely this expression multiplied by a constant, representing the ratio of droplets to bubbles, and therefore in the current paper we will address the process efficiency via the following expression:

$$
\eta \equiv \frac{W_{\text {surface } @ \tau}}{W_{p}}=\frac{\sigma\left(\frac{A_{b}}{V_{l}}\right)_{@ \tau}}{p\left(\frac{V_{b}}{V_{l}}\right)_{@ \tau}}=\frac{\sigma \frac{4 \pi}{3} \dot{n} \dot{r}^{2} \tau^{3}}{p \varepsilon}=\frac{\sigma \frac{4 \varepsilon}{(\dot{r} \tau)}}{p \varepsilon}=\frac{\sigma}{p \cdot(S M D)_{b}}
$$

\section{Results and Discussion}

The container pressure is close to the spinodal pressure, thus, if we limit ourselves to temperatures for which the pressure at the spinodal is negative, then the pressure difference between the spinodal and its respective saturation pressure can be practically represented by the spinodal pressure itself. Such large pressure differences produce high bubble growth rates. A dimensional analysis shows that even under these conditions, the surface tension and viscous effects on the bubble growth rate are negligible as compared to the inertial one, 
and the liquid can be considered here as a weakly compressible fluid [14]. The bubble growth rate as a function of the spinodal pressure and respective temperature is presented in Figure 2.

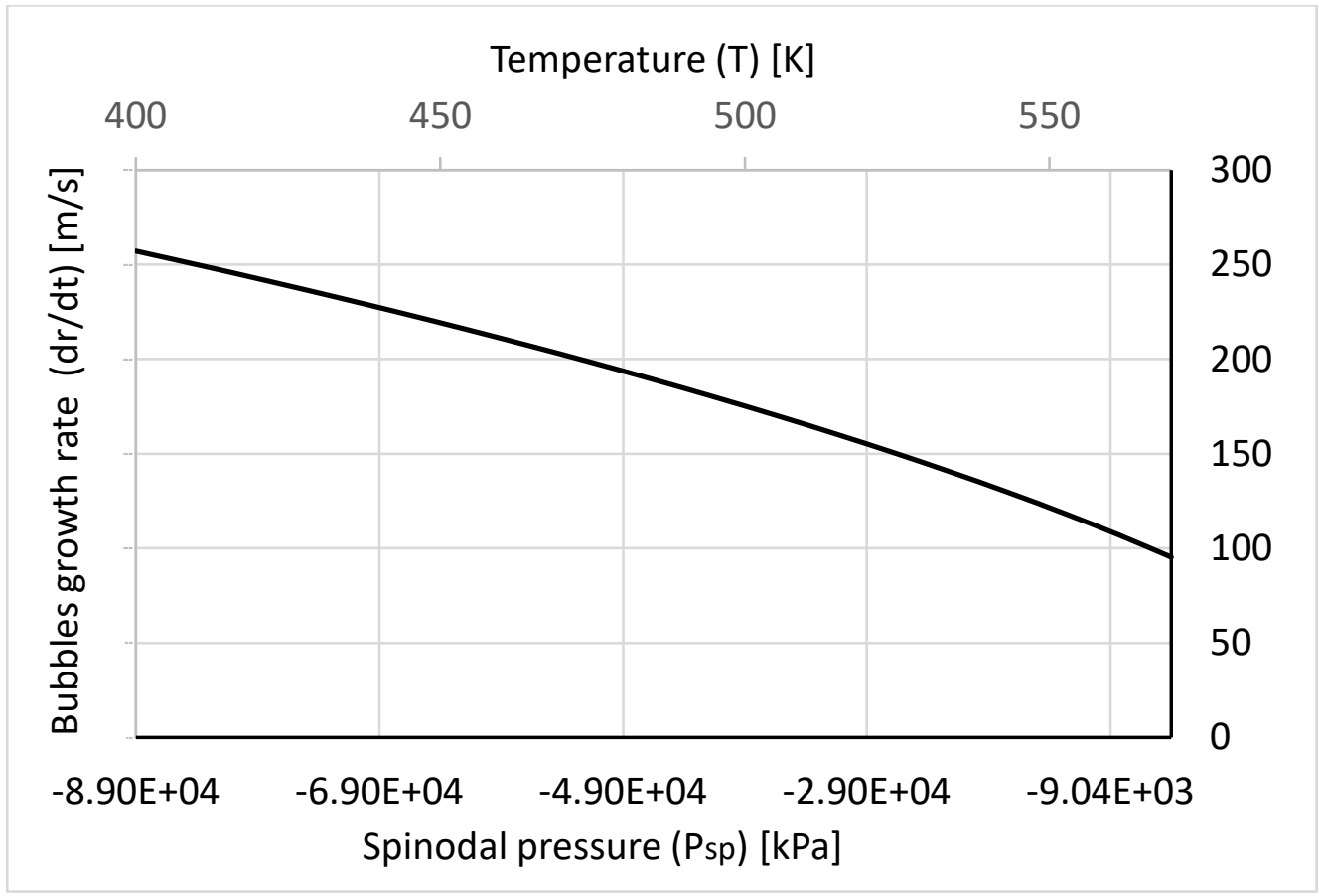

Figure 2. Bubbles' growth rate vs. spinodal pressure and respective temperature for $\mathrm{H}_{2} \mathrm{O}$.

For a given nucleation rate, higher bubbles growth rates mean shorter time durations until the grown bubbles touch each other (time to burst, see Figure 3). Close to the spinodal, the uncertainties in the nucleation rates become large, and thus it is interesting to see how does the nucleation rate affect the time to burst. As expected, higher nucleation rates promote shorter times until the bubbles touch each other.

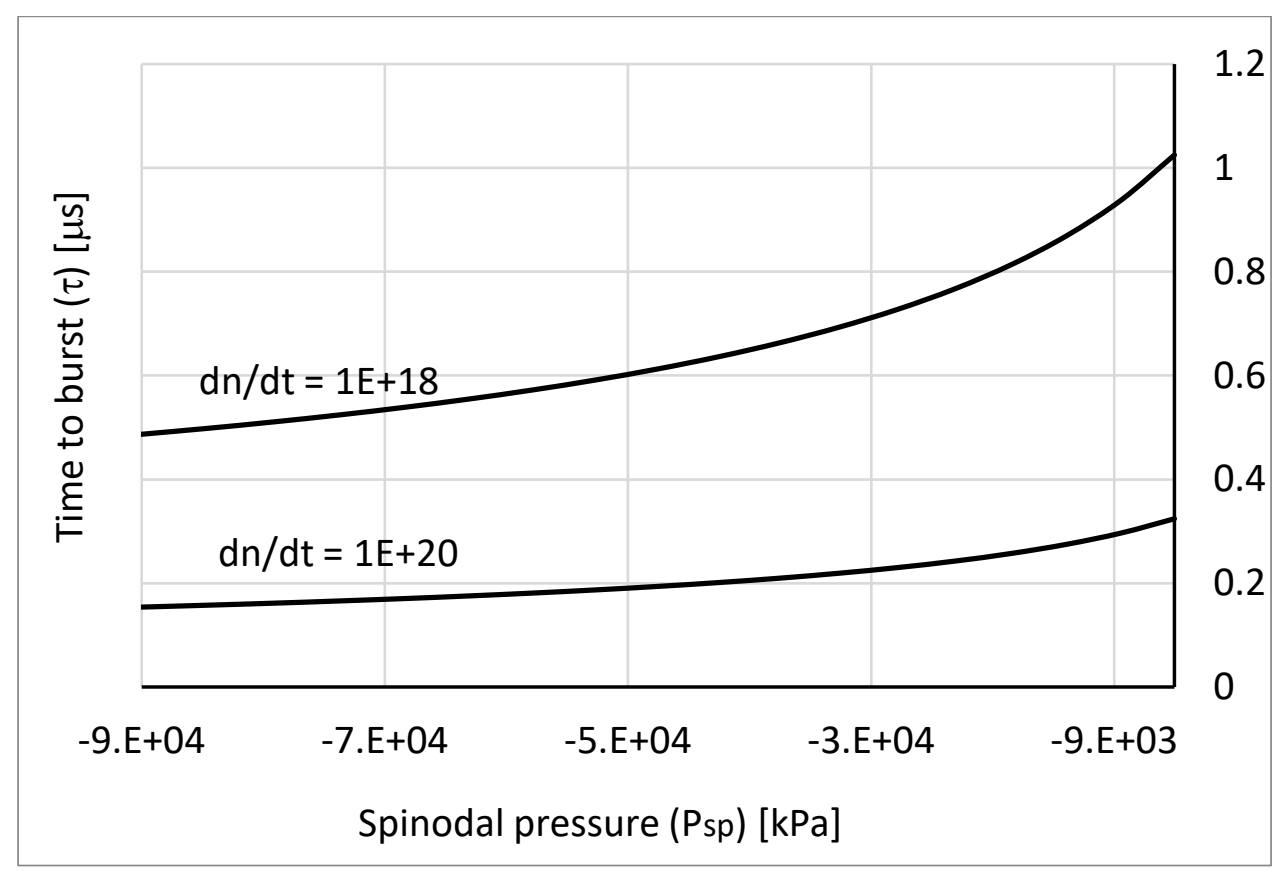

Figure 3. Time to burst vs. spinodal pressure for two different nucleation rates. 
Figures 2 and 3 suggest two opposing trends: on the one hand, the bubbles' growth rate increases with the increase in pressure difference, and on the other hand the time to burst decreases. For the SMD, the increase in the bubble growth rate is more dominant, and thus the bubbles' mean diameter is larger as the pressure difference becomes larger (see Figure 4). The atomization efficiency is therefore decreased, as clearly seen in Figure $\mathbf{5}$. This trend is counter-intuitive, as we would have expected that as the driving force for bubble growth increases, the pressure difference increases, and thus the atomization process will be more efficient. However, it seems that reduction in the duration of the process (time to burst), is dominant, and thus, the efficiency is deteriorated.

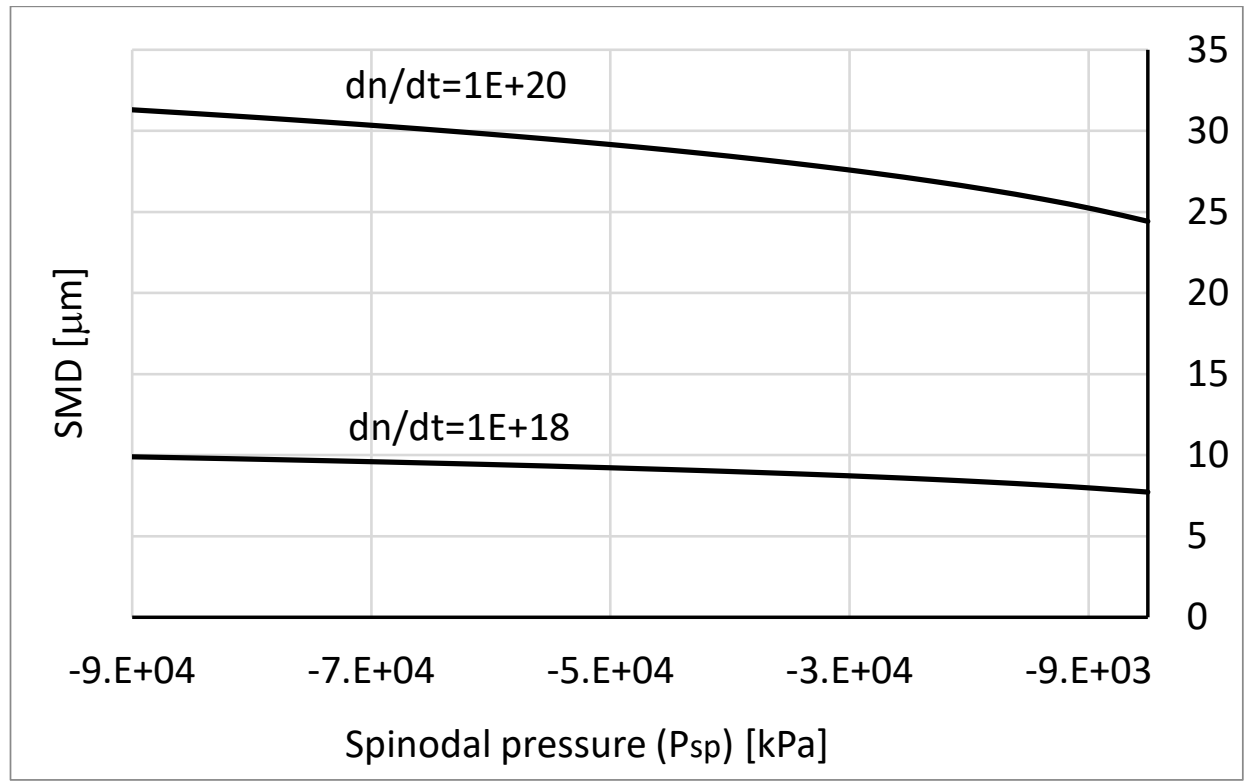

Figure 4. SMD vs. spinodal pressure for two different nucleation rates.

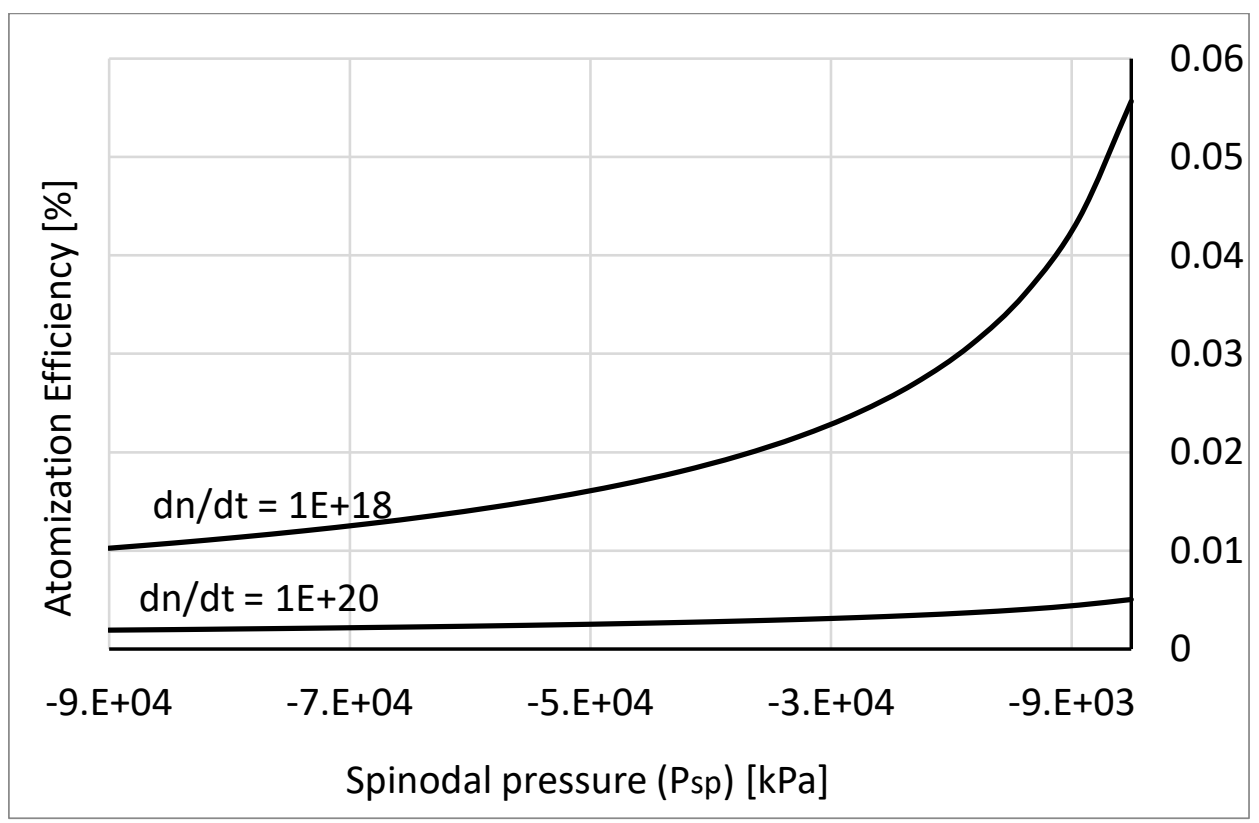

Figure 5. Atomization efficiency vs. spinodal pressure for two different nucleation rates. 


\section{Summary}

Flash boiling atomization process of a liquid under tensile conditions is considered. Here we examine a hypothetical process of constant pressure in order to examine the energetic profitability of the maximal theoretical pressure difference with respect to the spray formation.

It was found that for a given nucleation rate, the atomization efficiency deterioratess (rather than improved). This outcome is counter-intuitive, as we would expect that increasing the driving force for bubble growth, higher efficiency would be obtained. This counter-intuitive behavior could be explained by the fact that the bubble growth rate is so large, so at the time the bubbles touch each other, i.e. the time to burst, the bubbles are very large, so that the number of droplets that will be formed as a result of this rapid growth is decreased, rather than increased.

This mechanism might explain other experimental results, not necessarily pertaining to liquid under tension, where it was found that for homogeneous nucleation, as is considered here, higher pressure results in a higher droplet size [15].

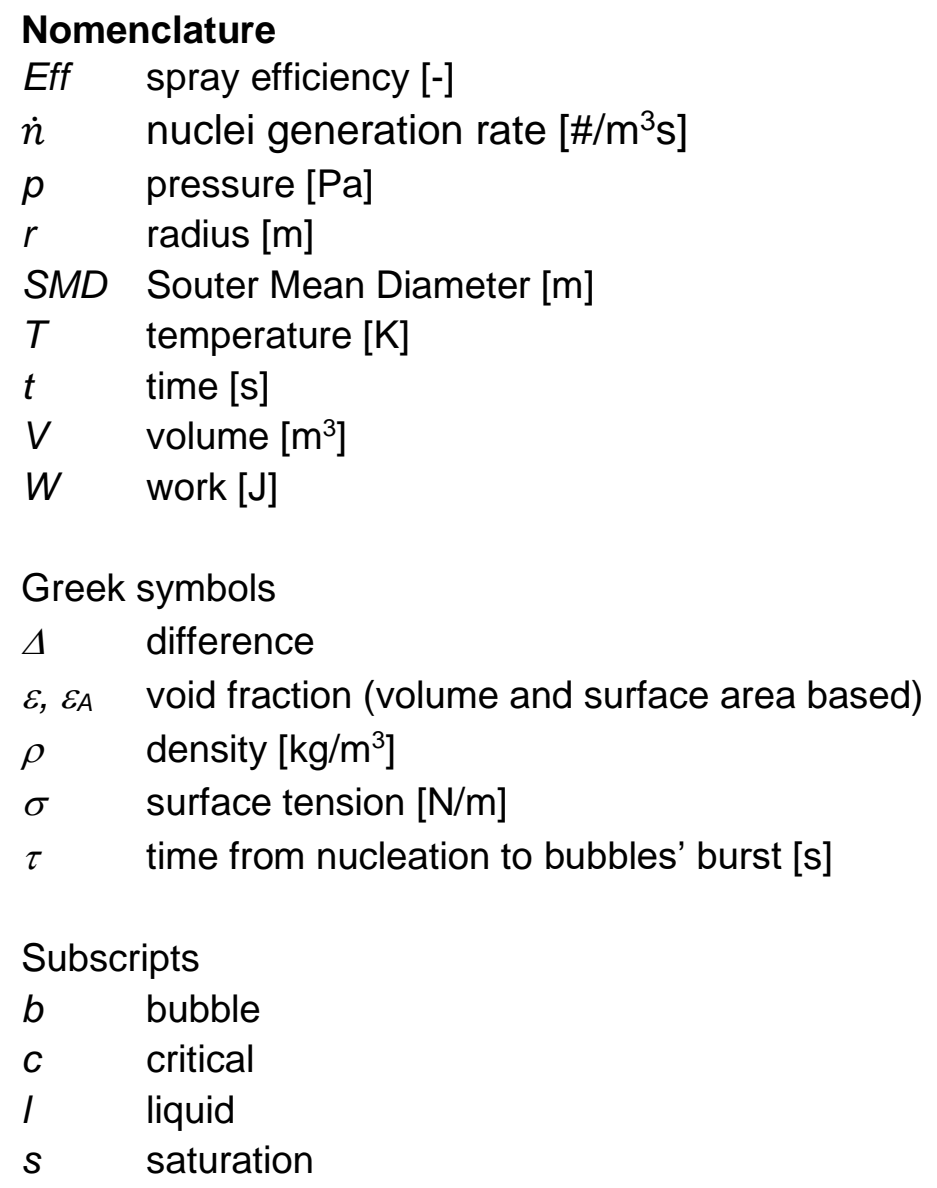

\section{References}

[1] Caupin, F., and Stroock, A.D., 2013, Advances in Chemical Physics, 152, 51-80.

[2] Wheeler, T.D., and Stroock, A.D., 2008, Nature, 455, 208.

[3] Karshafian, R., Bevan, P.D., Williams, R., Samac, S., and Burns, P.N., 2009, Ultrasound in medicine \& biology, 35, 847-860.

[4] www.chem.libretexts.org. 
[5] Caupin, F., Arvengas, A., Davitt, K., Azouzi, M. E. M., Shmulovich, K. I., Ramboz, C., \& Stroock, A. D., 2012, J Physics: Condensed Matter, 24, 284110.

[6] Briggs, L.J., 1951, J Chemical Physics, 19.7, 970-972.

[7] Bar-Kohany, T., and Sher, E., Short letter, Accepted for publication in Atomization and Sprays

[8] Skripov, V. P., 1974, "Metastable liquids." Wiley.

[9] Sher, E., and Elata, C., 1977, Industrial \& Engineering Chemistry Process Design and Development, 16, 237-242.

[10] Loureiro, D.D., Reutzsch, J., Kronenburg, A., Weigand, B. and Vogiatzaki, K., 2020, International J of Multiphase Flow, 132, 103405.

[11] Bar-Kohany, T. and Levy, M., 2016, Atomization and Sprays, 26, 1259-1305.

[12] Moshkovich, Y., Levy, Y., and Sher, E., 2019, Chemical Engineering Science, 206, 471-475.

[13] Carey, V. P., 2020, "Liquid-vapor phase-change phenomena: an introduction to the thermophysics of vaporization and condensation processes in heat transfer equipment." CRC Press.

[14] Bar-Kohany, T, Haustein, H, and Elias, E, July. 22-26 2018, 14th International Conference on Liquid Atomization and Spray Systems, ICLASS-14.

[15] Moshkovich, Y, Levy, Y, and Sher, E, 2021, International J of Multiphase Flow, 134, 103476. 